\title{
Progression towards Heart Failure after Myocardial Infarction Is Accompanied by a Change in the Spatial QRS-T Angle
}

\author{
Marjolein C de Jongh ${ }^{1}$, Agnese Sbrollini ${ }^{2}$, Arie C Maan ${ }^{1}$, Enno T van der Velde ${ }^{1}$, Martin J Schalij ${ }^{1}$, \\ Cees A Swenne ${ }^{1}$ \\ ${ }^{1}$ Leiden University Medical Center, Leiden, The Netherlands \\ ${ }^{2}$ Università Politecnica delle Marche, Ancona, Italy
}

\begin{abstract}
Myocardial infarction (MI) is a major risk factor for heart failure (HF). The ECG is known to change during the acute and the healing phases of MI, and it stabilizes in the chronic phase. We expect that further ECG changes in the chronic period actually signify a worsening clinical condition / emergence of HF. One major characteristic that signifies an ECG change is the spatial QRS-T angle (SA). It is generally believed that a worsening ECG is accompanied by an enlargement of $S A$, but we have seen that some patients who developed $H F$ after MI showed a decrease of SA. In the current study we aim to demonstrate that development of HF after MI is accompanied by a change in SA, but irrespective its sign.

We retrospectively studied 81 control patients (67/14 male/female, mean $\pm S D$ age $64 \pm 10$ years) and 48 cases (38/10 male/female mean $\pm S D$ age $60 \pm 12$ years). Control patients had a healed MI and had, thereafter, two elective ECGs (baseline and follow-up) made $>1$ year apart. Case patients had a healed MI but presented later at our $H F$ outpatient clinic with HF symptoms. Their baseline ECG was made at least 6 months post MI; the ECG made when presenting at the HF outpatient clinic was called followup ECG. In each ECG, we computed $S A$, and in each patient we computed the difference between the baseline and follow-up SAs. Finally we performed a ROC analysis on the signed and unsigned individual SA differences.

ROC analysis revealed an area-under-the-curve (AUC) of 0.71 for signed SA differences, and 0.78 for unsigned SA differences. Both AUCs were significantly larger than 0.5, but the difference between these two curves did not reach statistical significance.

In conclusion our study suggests that the best casecontrol separation can be attained by considering absolute SA changes, but a larger study is needed to demonstrate that $S A$ changes irrespective the sign are performing significantly better than signed SA changes. The absolute change in $S A$ is a promising ECG feature to detect emerging $H F$ in post-MI patients.
\end{abstract}

\section{Introduction}

Heart failure (HF) is a growing epidemic that opposes a large health and financial burden to the society. Once diagnosed, the 5-year survival estimates are between 40$50 \%$ [1]. Although the incidence of HF on the basis of non-ischaemic causes, often with preserved ejection fraction, is increasing, more than $50 \%$ of patients with $\mathrm{HF}$ have a history of MI[2]. Early detection and treatment of HF can reduce the extent of myocardial remodelling and improves the prognosis[3]. Our current research is intended to investigate if periodic ECGs can play a role in the early detection of impending HF.

The ECG is known to change during the acute and the healing phases of MI; it stabilizes in the chronic phase of MI. We expect that further ECG changes in the chronic phase actually signify a worsening clinical condition / emergence of HF. One major variable that signifies an ECG change is the spatial QRS-T angle (SA). It is generally believed that a worsening ECG is accompanied by an enlargement of SA, but we have seen that some patients who developed HF after MI showed actually a decrease of SA. In the current study we aim to demonstrate that development of $\mathrm{HF}$ after $\mathrm{MI}$ is accompanied by a change in SA, but irrespective its sign.

\section{Methods}

In this study we compared a control and a case group. The control group was a subset of patients studied by Treskes et al[4]. These patients were retrospectively found in the electronic patient database (EPD) of the Leiden University Medical Centre (LUMC). Selection criteria were:

- Healed MI in the patient history

- Availability of two elective digital standard 12-lead ECGs made approximately 1 year apart, called baseline ECG (BL-ECG) and follow-up ECG (FU-ECG)

- No development of HF and clinically stable between the BL-ECG and FU-ECG. 
The case group was specially collected for the current study. These patients were also found retrospectively in the EPD of the LUMC. Selection criteria were:

- Healed MI in the patient history

- Development of a clinically stable period without any sign of HF within 6 months from MI

- Development of HF after this stable phase

- Availability of two elective digital standard 12lead ECGs; the first available ECG in the stable period was used as BL-ECG and the first ECG made at the initial presentation with $\mathrm{HF}$ was used as FU-ECG

- No major cardiac event in the period between these two ECGs

HF is based on a clinical diagnosis that rests on careful evaluation of a multitude of symptoms, results of function tests, imaging and laboratory data[5]. The ejection fraction $(\mathrm{EF})$ was not considered in the diagnosis of $\mathrm{HF}$, as there are patients with a reduced $\mathrm{EF}$ without $\mathrm{HF}$ as well as patients with preserved EF with HF.

Patients with a major cardiac event between BL-ECG and FU-ECG were not included in the case and control group. PCI and CABG were not considered as major cardiac events when performed electively. The DOR procedure was considered as a major cardiac event; patients with this operation were not included in the study. Only patients in whom both the BL-ECG and the FU-ECG showed sinus rhythm were included.

The ECGs were processed by our Matlab program LEADS[6]. This program performs a vectorcardiographic ECG analysis based on a synthesized vectorcardiogram (VCG). VCG synthesis from the 12-lead ECG was done by using the Kors matrix[7]. SA was computed as the planar angle between the QRS axis and the T axis (i.e., the orientations of the spatial QRS- and T integrals).

We computed for all patients the difference $\triangle \mathrm{SA}$ between the SA in the FU-ECG and the SA in the BLECG; positive $\triangle \mathrm{SA}$ indicates an increase and negative $\triangle \mathrm{SA}$ a decrease of SA in the patient.

Statistical analysis was done for $\Delta \mathrm{SA}$ and for $|\Delta \mathrm{SA}|$. Case-control differences for $\Delta \mathrm{SA}$ and for $|\Delta \mathrm{SA}|$ were analysed by a Wilcoxon test. Finally, the diagnostic performance of $\Delta \mathrm{SA}$ and of $|\Delta \mathrm{SA}|$ was evaluated by receiver-operating-characteristic (ROC) analysis.

\section{Results}

The study group comprised 48 cases and 81 controls, see Tables 1 and 2. Figures 1 and 2 show the distributions of the SA differences between the baseline and follow-up ECGs in the cases and in the controls. Figure 1 shows the signed differences and Figure 2 the absolute values of the differences.
Table 1. Patient characteristics, time between BL- and FU-ECG, and spatial angle (SA) in the BL-ECG (cases).

\begin{tabular}{|c|c|c|}
\hline Cases: $\mathrm{N}=48$ & Mean \pm SD & Range \\
\hline $38 / 10$ male/female & & \\
\hline Age at inclusion (y) & $60 \pm 12$ & $31-78$ \\
\hline $\begin{array}{l}\text { Time between BL-ECG } \\
\text { and FU-ECG (y) }\end{array}$ & $7 \pm 5$ & $1-22$ \\
\hline SA of BL-ECG $\left(^{\circ}\right)$ & $106 \pm 36$ & $29-170$ \\
\hline
\end{tabular}

Table 2. Patient characteristics and spatial angle (SA) in the BL-ECG (controls). Time between BL- and FU-ECG was approximately 1 year.

\begin{tabular}{lll}
\hline Controls: $\mathrm{N}=81$ & Mean \pm SD & Range \\
\hline 67/14 male/female & & \\
Age at inclusion (y) & $64 \pm 10$ & $37-92$ \\
SA of BL-ECG $\left({ }^{\circ}\right)$ & $92 \pm 40$ & $17-176$ \\
\hline
\end{tabular}

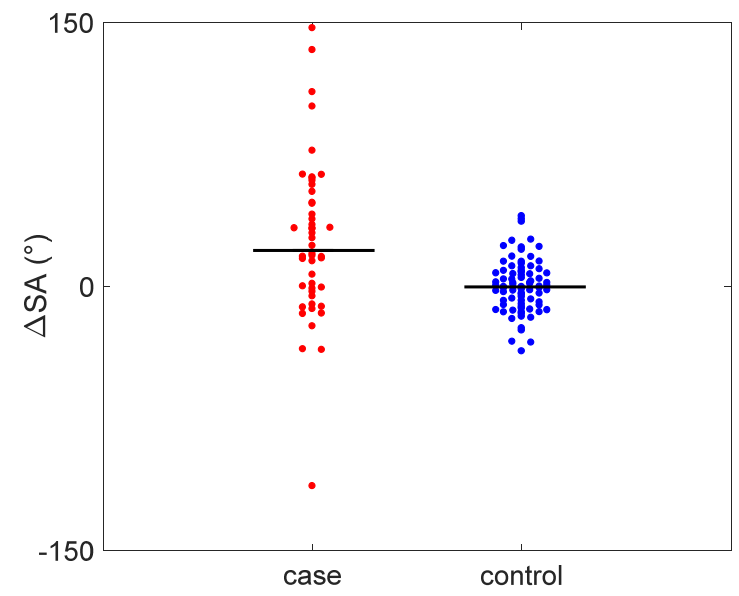

Figure 1. Spread plot of the $\Delta \mathrm{SA}$ in the cases (red) and in the controls (blue). The black horizontal lines are the medians of these groups.

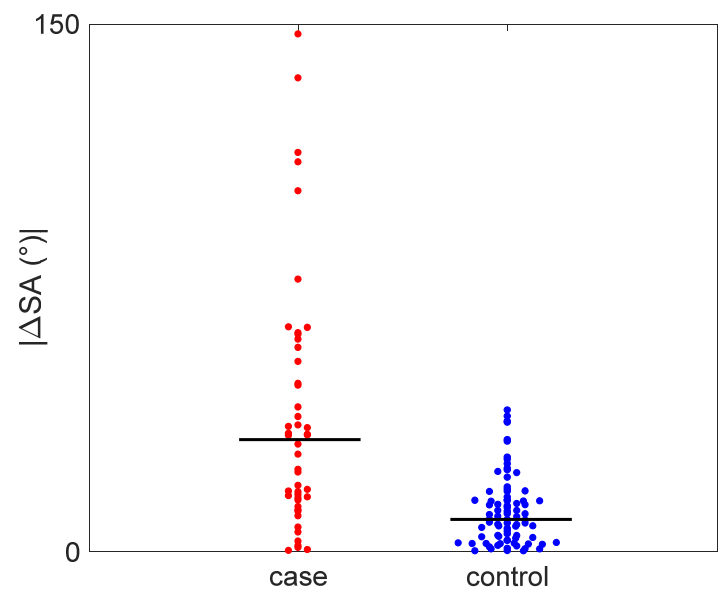

Figure 2. Spread plot of the $|\Delta \mathrm{SA}|$ in the cases (red) and in the controls (blue). The black horizontal lines are the medians of these groups. 
$\triangle \mathrm{SA}$ was not normally distributed. For this reason we compared the median values of the cases and the controls by Wilcoxon tests. The medians of the case and control patients differed significantly in the signed as well as the absolute value data, see Table 3 .

Table 3. Results of the statistical comparison of the medians of $\Delta \mathrm{SA}$ and $|\Delta \mathrm{SA}|$ by Wilcoxon tests.

\begin{tabular}{llll}
\hline & Cases & Controls & $P$ value \\
\hline$\Delta$ SA $\left(^{\circ}\right)$ & 20.99 & 0.11 & $<0.001$ \\
$|\Delta \mathrm{SA}|\left(^{\circ}\right)$ & 31.70 & 9.06 & $<0.001$ \\
\hline
\end{tabular}

Figures 3 and 4 show the ROCs for $\triangle \mathrm{SA}$ and $|\Delta \mathrm{SA}|$, respectively. The areas under the curve (AUC) were significantly larger than 0.5 . The area of the ROC for $|\triangle \mathrm{SA}|(0.78)$ was larger than the area of the ROC for $\triangle \mathrm{SA}$ (0.72), but this difference did not reach statistical significance.

\section{Discussion}

In this study we demonstrated that changes in the spatial angle (SA) can be used to discriminate patients who develop HF after MI (cases) and patients who do not (controls). Also, the results of our study suggest that our initial hypothesis, that not only increases in SA but also decreases in SA can signify emergence of $\mathrm{HF}$, is potentially correct. Evidence for this is the larger AUC of the ROC for $|\triangle \mathrm{SA}|(0.78)$ than the AUC of the ROC for $\triangle$ SA (0.72). In our view, the fact that this difference did not reach statistical significance is caused by the limited size of our study group.

When inspecting the shapes of the ROCs, it is striking that the ROC of $|\Delta \mathrm{SA}|$ has a much more ideal shape then the ROC of $\triangle \mathrm{SA}$, in which roughly at $60 \%$ sensitivity the specificity becomes dramatically worse. The shape of the ROC of $|\Delta \mathrm{SA}|$ shows a steadily changing trade-off between sensitivity and specificity over its full range.

Our study provides an argument for a different interpretation of the spatial angle as traditionally assumed. Usually, an increase in SA, and, hence, a decrease in concordance of the ECG, is associated with pathological processes. Several forms of cardiac pathology are associated with enlarged spatial angles. However, when patients are already known with existing cardiac pathology (MI in our study group), they have already an enlarged SA (see Tables 1 and 2). If then further pathology develops, it depends on the location in the heart where the largest pathological alterations occur what happens with the QRS and the T axes. Eventually, the new pathology can, in the follow-up ECG, partly neutralize the change in SA that had occured due to the initially existing pathology. Actually, this can be considered as a form of pseudo-normalization. Hence, we must interpret any change in SA as emerging pathology, regardless of the sign.

The quality of the discrimination of cases and controls as represented by the ROC of $|\triangle \mathrm{SA}|$ in Figure 4 can be characterized as fair-to-good[8]. This renders serial ECG in patients with a healed myocardial infarction a potential instrument for periodic screening of these patients with the purpose of early detecion of possible emerging heart failure.

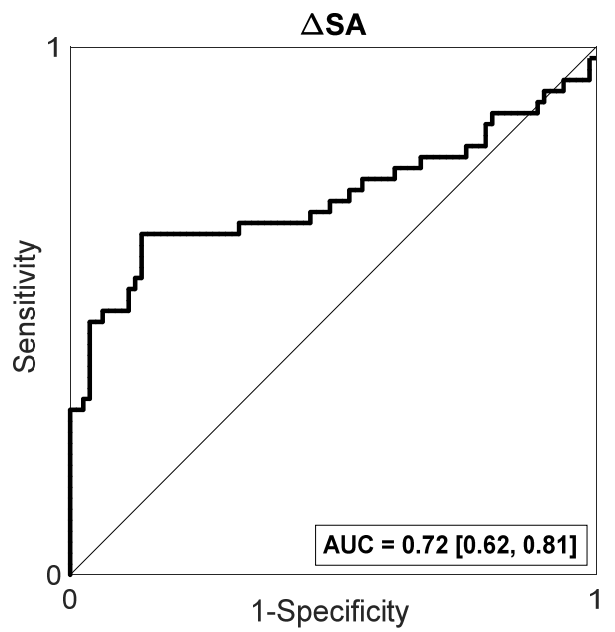

Figure 3. Receiver-operating characteristic (ROC) for the discrimination of cases and controls on the basis of $\Delta \mathrm{SA}$. $\mathrm{AUC}=$ area under the curve. Values between square brackets are the $95 \%$ confidence intervals.

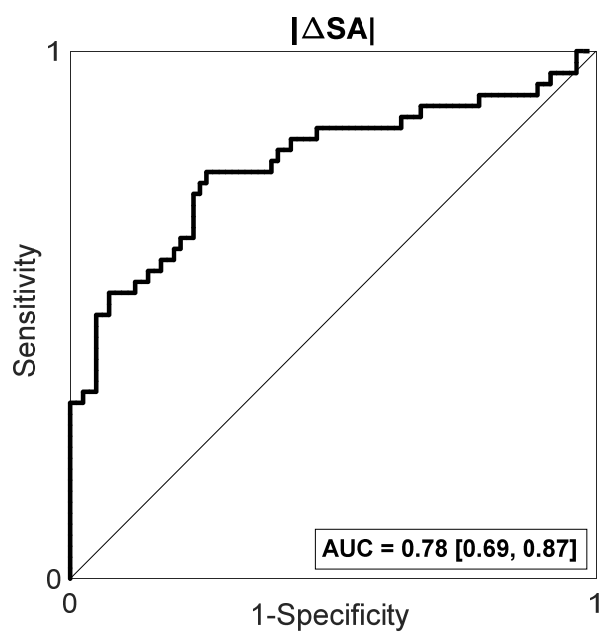

Figure 4. Receiver-operating characteristic (ROC) for the discrimination of cases and controls on the basis of $|\Delta \mathrm{SA}|$. $\mathrm{AUC}=$ area under the curve. Values between square brackets are the $95 \%$ confidence intervals. 


\section{References}

[1] Benjamin EJ, Blaha MJ, Chiuve SE, Cushman M, Das SR, Deo R, et al. Heart Disease and Stroke Statistics-2017 Update: A Report From the American Heart Association. Circulation. 2017;135(10):e146-e603.

[2] Mosterd A, Hoes AW. Clinical epidemiology of heart failure. Heart. 2007;93(9):1137-46.

[3] Bhatt AS, Ambrosy AP, Velazquez EJ. Adverse Remodeling and Reverse Remodeling After Myocardial Infarction. Curr Cardiol Rep. 2017;19(8):71.

[4] Treskes RW, Ter Haar CC, Man S, De Jongh MC, Maan AC, Wolterbeek R, et al. Performance of ST and ventricular gradient difference vectors in electrocardiographic detection of acute myocardial ischemia. Journal of electrocardiology. 2015;48(4):498-504.

[5] Ponikowski P, Voors AA, Anker SD, Bueno H, Cleland JG, Coats AJ, et al. 2016 ESC Guidelines for the diagnosis and treatment of acute and chronic heart failure: The Task Force for the diagnosis and treatment of acute and chronic heart failure of the European Society of Cardiology (ESC)Developed with the special contribution of the Heart Failure Association (HFA) of the ESC. European heart journal. 2016;37(27):2129-200.

[6] Draisma HHM, Swenne CA, van de Vooren H, Maan AC, Hooft van Huysduynen B, van der Wall EE, et al. LEADS: An Interactive Research Oriented ECG/VCG Analysis System. Computers in Cardiology. 2005;32:515-8.

[7] Kors JA, van Herpen G, Sittig AC, van Bemmel JH. Reconstruction of the Frank vectorcardiogram from standard electrocardiographic leads: diagnostic comparison of different methods. European heart journal. 1990;11(12):1083-92.

[8] Tape TG. Interpretation of diagnostic tests. Ann Intern Med. 2001;135:72.

Address for correspondence:

Cees A. Swenne, $\mathrm{PhD}$

Cardiology Department

Leiden University Medical Center

PO Box 9600, 2300 RC Leiden, The Netherlands

c.a.swenne@lumc.nl 